It will be noticed that in none of these cases is there unquestionable proof of the absorption of altogether dead bone. The increase of the ruddy blush shining through the sequestrum may be in any such case the result of increased vascularity, subjacent to an originally thin sequestrum. But, in the previously described case, the proof was completed by perforation and diminution in superficial area of the exfoliation. In all four cases, permanganate of potash happened to be the only application used.

\section{NOTES ON TUMOURS.}

By THOMAS ANNANDALE, F.R.S.E., F.R.C.S.Ed. Lecturer on Surgery, and Assistant-Surgeon to the Edinburgh Royal Infirmary.

THE following notes are the result of the last eight years' observation and study of a considerable number and variety of cases of tumour, met with principally in the hospital and private practice of Mr. Syme, who kindly permits me to make use of them.

\section{FATTY TUMOURS.}

Painful Symptoms. - Fatty tumours would appear to cause pain in, and even weakness of, a part more frequently than is generally supposed, for, since Mr. Syme first directed my attention to the fact, I have met with many cases of the kind during the last few years. In the majority of these cases, the patients have been females, and the tumours situated on the shoulder, back, or upper extremity. The pain complained of was of a darting or shooting character, was not usually constant, was occasionally accompanied by weakness of neighbouring muscles, and was entirely relieved by the removal of the tumour. Dissection of the tumour removed did not show any peculiarity of structure or special nervous connection. In one interesting case (Case $\mathrm{x}$ ), the tumours were multiple.

The following is a short report of some of these cases.

CASE I. - A woman, aged 25, had a small fatty tumour of the size of a marble situated over the right coracoid process. The patient suffered from pain in the tumour and shooting down the arm. There was also weakness of the whole arm, which prevented her from following her oc cupation as a sempstress. The removal of the tumour entirely relieved the pain and weakness.

CASE II. - A man, aged 44, had a small fatty tumour on the left side of his neck. He suffered from pain commencing in the tumour and shooting down the neck towards the shoulder. Excision of the tumour quite relieved these symptoms.

CASE III.-A woman, aged 38 , had a fatty tumour of the size of an orange over the apex of the left scapula. She had pain in the tumour, pain shooting down the corresponding arm, and also weakness of the same limb. When the tumour was cut out, these symptoms disappeared.

CASE IV. - A woman, aged 60 , had a fatty tumour of the size of a hen's egg over the right clavicle, which caused shooting pains in the corresponding arm. Removal of the tumour cured the pain.

CASE v.-A woman, aged 47, had a small fatty tumour in front of the right shoulder, which caused shooting pains in, and great weakness of, the corresponding arm. The tumour was removed, and the pain and weakness were relieved.

CASE VI.-A woman, aged 40, had a fatty tumour of the size of a pigeon's egg over her left scapula. Her left arm was weak, and she frequently suffered from pain shooting down the corresponding arm as far as the elbow. Excision of the tumour cured these symptoms.

CASE VII. - A woman, aged 40, applied for advice on account of pain in her right shoulder and arm, the result, as she supposed, of a recent bruise. On examination, one small fatty tumour was found in front of the joint, and two others on its outer aspect. On careful imquiry, it appeared that she had suffered from the painful symptoms long before she received the injury, so that the tumours were evidently the real cause of the pain.

CASE VIII. - A woman, aged 40, had a fatty tumour of the size of a small saucer over the dorsal region. For the last two years, the tumour had caused pain running down her back, round the chest to her left breast, and up towards her left shoulder. She had also a feeling of weakness in the back. The removal of the tumour cured these symptoms.

CASE IX. - A woman, aged 38 , had a fatty tumour of the size of a small plate over the outer aspect of her right arm, a little below the shoulder. The tumour had existed seven years. About three months ago, the patient commenced to suffer from pain shooting down the arm and passing into the ball of the thumb, which often felt numb. The tumour was cut out, and these symptoms were removed.

CASE X.-In May 1864, a woman, aged 48, applied for advice in regard to three small tumours on the anterior aspect of her right forearm. These tumours caused her much pain, which passed down to the thumb and index-finger. The patient had three similar tumours in the corresponding situation of her left forearm, which also caused painful symptoms. All the tumours were removed from both forearms, with the effect of curing the pain. In January 1865 , the patient returned, with three new tumours of the same nature in the right arm, and several others in the forearm. She stated that the painful symptoms came back about three months after the last operation and have remained ever since. The tumours were removed, and the symptoms again disappeared. In April I866, the patient returned a third time with similar tumours in her arm and forearm, which were accompanied by the painful symptoms. The tumours removed from this patient were firm in consistence, slightly lobulated, and varied in size from a pea to a small nut.

Lobes or Processes. - Pendulous fatty tumours have frequently lobes or processes spreading under the skin for a greater or less distance from their point of attachment to it. In removing such a tumour, it is not sufficient to cut across its neck; but the processes, which form as it were a root, must be carefully dissected out. In order to illustrate this, I have figured a pendulous fatty tumour which was removed from the thigh of a lady aged 45. (Fig. I.) The tumour was a little larger than a turkey's

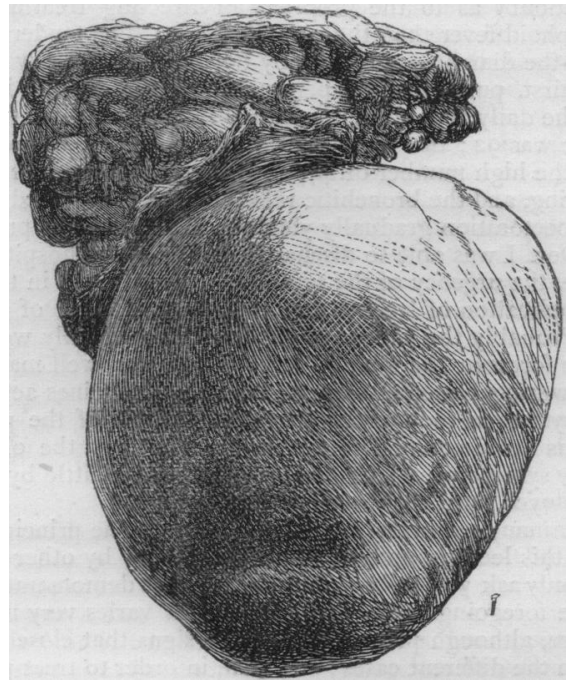

egg; it had a distinct neck, and a root consisting (as is shown in the woodcut) of numerous lobules or processes, which were dissected out in the removal of the tumour.

Fig. 2 illustrates a case of fatty tumour which had a lobule or process

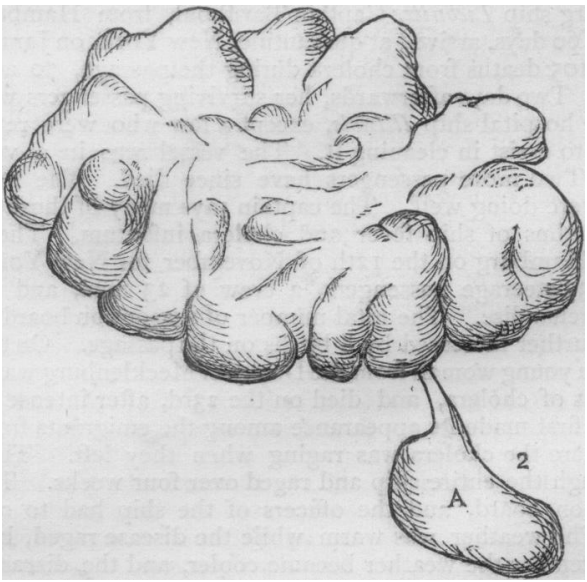

(A) connected to it by a long narrow neck. This tumour was a simple lobulated fatty tumour situated on the back of a gentleman aged 50. In removing the tumour, the process was found passing underneath the skin; it was distant from the mass of the tumour itself fully an inch and a half.

Rarer Situations. - I have met with three cases of fatty tumour situated on the forehead. In all these cases, the tumours were small, not much 
lobulated, and somewhat adherent to the skin. A case of fatty tumour situated over the right parotid gland lately came under my notice. It was of the size of a hen's egg, and presented no peculiarity, except that after removal it had a curious glistening appearance, as if it had been subjected to heat.

Fig. 3 is taken from a case of fatty tumour lying underneath a muscle. I met with it in dissecting a female subject. The tumour was the size of a hen's egr, and was lying underneath the tensor fascix femoris muscle. The muscular filves were stretched over the tumour, which.was closely adherent to them, and was surrounded by the same layer of fascia which invested the muscle. In the woodcut, the under surface of the muscle and tumour is shown.

For the following interesting case of fatty tumour simulating an inguinal hernia, $I$ am indebted to Dr. Gillespie, who kindly allowed me to examine the patient.

J. H., aged 26, was admitted into the Royal Infirmary, under the care of Dr. Gillespie, with a tumour occupying the right groin and labium. Two years ago, she first noticed a swelling in this region, and applied to a surgeon for advice. She was told it was a "rupture", and was ordered a truss. This instrument having no effect in relieving the swelling, she was recommended to come to the hospital. On examination, there was a lobulated tumour of the size of a closed fist, lying partly in the right labium and partly in the right inguinal region. The swelling did not extend higher than the external abdominal ring; it had no neck, and did not receive any impulse when the patient coughed. The tumour was removed with perfect success, and was found to be an ordinary lohulated fatty one.

Degenerations. - In examining fatty tumours

of a large size, or which have existed for a long time, I have frequently seen portions of their structure altered in consistence and colour, espe. cially in cases where the tumour had been subjected to pressure or irritation. In two cases of large fatty tumour, I found a small portion of their tissue condensed and of a greenish hue. The two following cases are examples of calcareous degeneration of fatty tumours, a condition by no means common.

CASE I. - M. S., aged 53, was seen in July 1862 with a fatty tumour situated at the lower border of the right axilla. The tumour was of the size of a small saucer, had existed nine years, and was quite move. able. It was cut out by Mr. Syme; and, in dissecting it, I found in its centre a calcareous /mass of the size of a large filbert. This calcareous substance, when cut, resembled very much in appearance cheesy tubercular matter, but it was harder and more gritty.

CASE II. - R. M., aged 40, was seen in December 1866 with a large fatty tumour situated on the outer aspect of the upper third of the left thigh. The tumour was of the size of a child's head, which had existed for twenty years, and was adherent to the skin by its anterior surface. The tumour was removed, and, on dissecting it, I lound a small calcareous mass as large as a finger-nail in its substance. This calcareous mass resembled in appearance and structure that met with in the former instance. In neither of these cases was there any other peculiarity in the structure of the tumours.

\section{RESEARCHES ON THE ACTION OF THE HEART.*}

\section{By GEORGE PATON, M.D., Bowmanville, Canada West.}

MORE fully to explain our views, we may observe that, having denuded the heart of a living turtle, we carefully observed the phenomena. Respiration remained unaffected. When the heart beat at ten or twelve pulsations per minute, the ventricle, after contraction, dilated, and became partially filled with blood passing into it from the distended auri. cles; a pause ensued; and then the auricles contracted, and completed the diastole of the ventricle, causing it to contract. When the action increased to sixteen or eighteen pulsations per minute, the ventricle,

- Read before the Physiological Section at the Annual Meeting of the British Medical Aseociation in Dublin, August 1867. [Continued from p. 120.] after contraction, dilated, and a portion of blood passed from the distended auricles into the ventricle, flling out its parietes; but, before this was completed, the auricles contracted, and, projecting their blood into the ventricle, brought it into a state of distension at once, which annihilated the pause, and rendered the diastole one continuous act. But, on the pulsa. tions increasing to 24 or 26 per minute, the auricles contracted synchronously with the diastole of the ventricle, which consisted of one continuous and uninterrupted movement. The blood projected by the auricles filled out or dilated the ventricle, as its walls expanded, till distension ensued and contraction took place. Here we observed that, as the diastole proceeded, a sort of undulation passed along the cavity of the ventricle, as if the blood, by the force which it cxerted, opened up and distended its walls. The animal remaining in a calm and quiescent state, and the action of the heart procecding with much uniformity, suddenly, as if impelled by an impulse, the auricles began to contract with great energy, and projected the blood into the ventricle with in. creased velocity; and its parietes expanded at a proportionate ratio, and contracted in a similar manner. The vigorous contraction of the auricles appeared to open up the relaxing parietes of the ventricle, and caused them to dilate with greater velocity. These movements would continue for a time, and then the slower action of the heart would return.

In another turtle, the action of whose heart we examined during the highest temperature of the season (July Sth), it appeared that the contraction of the auricles exerted an influence on the dilatation of the ventricle; for, when the action of the heart had attained its greatest degree of speed- 36 pulsations per minute-the contracting power of the ventricle was sometimes apparently prolonged, as if from the vigour with which it acted ; and, whilst the ventricle continued in this contracting state, the auricles contracted; and the moment the blood entered the foramen of the ventricle, its parietes opened up, and continued to expand till they contracted. This fact was also apparent when the action of the heart was 30 pulsations per minute. We placed our fingers against the parietes of the ventricle, and we distinctly recognised that they did not become smooth and soft till the auricles began to contract; and, the moment that took place, the blood appeared to open up the contracting walls of the ventricle, or caused them to expand till contraction commenced.

There was now a great difference in the character of the movements both of the auricles and the ventricle. When the action of the heart was slow, as at 10 or 12 pulsations per minute, the auricles, on being dilated, continued distended with blood during the first stage of the diastole and the pause. But, when the action of the heart was vigorous, the auricles were dilating whilst the ventricle was contracting; and, as the auriclesimmediately contracted on being dilated, theblood entered the foramen of the ventricle at the moment its fibres were beginning to relax, and rapidly dilated its walls, or filled out the cavity of the ventricle till distension was produced and contraction commenced.

We denuded the heart of a living frog, and carefully observed its action. The pulsations were 64 per minute. Respiration remained perfect. The auricles, on being dilated, immediately contracted, and they contracted synchronously with the diastole of the ventricle; the parietes of the ventricle continuing to expand as the blood was projected by the auricles, till distension was produced and the ventricle contracted. During dilatation, the ventricle assumed a deep red colour; and the blood could be distinctly seen to enter its cavity as the parietes expanded and dilated. During contraction, the ventricle became small and pale, all the blood appearing to be expelled from its cavity. But, after contraction, there was no expansion of the parietes of the ventricle; and no blood entered its cavity till the auricles contracted. The animal continued to respire with vigour, and the action of the heart increased to 72-74 per minute. The movements were maintained in a similar manner, but the auricles contracted with greater rapidity; and, as they projected the blood into the ventricle, its parietes expanded at a proportionate ratio, and also more quickly contracted, projecting the blood with greater velocity along the aorta. After a few hours, the action of the heart fell to 30 per minute, and precisely the same movements were observed; the auricles, on contracting, passing as it were into the foramen of the ventricle; and the blood could be distinctly seen to enter its cavity whilst the walls of the ventricle expanded and dilated. But there was not the slightest approach to anything like the dilatation of the ventricle before the contraction of the auricles.

On clenuding the heart in warm-blooded animals, we have carefully observed its action, both by touching the ventricles with the hands, and steadily watching their movements with the eye; the pulsations being in some cases, as in large animals, as the calf, 64 per minute; in other cases, as in small animals, 80 , 90 , or 100 per minute. But we have never been able to detect anything like a pause during the diastole of the ventricles. In all these cases, the contraction of the auricles was synchronous with the diastole of the ventricles, which consisted of one 